\title{
In vivo Measurement of Knee Extensor Muscle Function in Mice
}

\author{
Camille R. Brightwell ${ }^{1,2}$, Ted G. Graber ${ }^{3}$, Benjamin D. Brightwell ${ }^{4,5}$, Matthew Borkowski ${ }^{6}$, Brian Noehren ${ }^{5,7}$, Christopher S. \\ Fry $^{1,2}$ \\ ${ }^{1}$ Department of Athletic Training and Clinical Nutrition, University of Kentucky ${ }^{2}$ Center for Muscle Biology, University of Kentucky ${ }^{3}$ Department of Physical \\ Therapy, East Carolina University ${ }^{4}$ Kinesiology and Health Promotion Graduate Program, University of Kentucky ${ }^{5}$ Biomotion Lab, College of Health \\ Sciences, University of Kentucky ${ }^{6}$ Aurora Scientific ${ }^{7}$ Department of Physical Therapy, College of Health Sciences, University of Kentucky
}

\section{Corresponding Author}

Christopher S. Fry

cfr223@uky.edu

\section{Citation}

Brightwell, C.R., Graber, T.G.,

Brightwell, B.D., Borkowski, M.,

Noehren, B., Fry, C.S. In vivo

Measurement of Knee Extensor Muscle

Function in Mice. J. Vis. Exp. (169),

e62211, doi:10.3791/62211 (2021).

\section{Date Published}

March 4, 2021

DOI

$10.3791 / 62211$

URL

jove.com/video/62211

\section{Abstract}

Skeletal muscle plasticity in response to countless conditions and stimuli mediates concurrent functional adaptation, both negative and positive. In the clinic and the research laboratory, maximal muscular strength is widely measured longitudinally in humans, with knee extensor musculature the most reported functional outcome. Pathology of the knee extensor muscle complex is well documented in aging, orthopedic injury, disease, and disuse; knee extensor strength is closely related to functional capacity and injury risk, underscoring the importance of reliable measurement of knee extensor strength. Repeatable, in vivo assessment of knee extensor strength in pre-clinical rodent studies offers valuable functional endpoints for studies exploring osteoarthritis or knee injury. We report an in vivo and non-invasive protocol to repeatedly measure isometric peak tetanic torque of the knee extensors in mice across time. We demonstrate consistency using this novel method to measure knee extensor strength with repeated assessment in multiple mice producing similar results.

\section{Introduction}

Skeletal muscle is a highly adaptable tissue with compensatory alterations to mass and structure in response to a myriad of stimuli, such as exercise, nutrition, injury, disease, aging, and disuse. Many studies investigating skeletal muscle adaption in humans employ methods to measure both skeletal muscle size and impact on function, as gold-standard strength assessments are easily repeatable in human subjects.

Specifically, knee extensor and flexor strength are most assessed in clinical research. Alterations to knee extensor strength have been widely reported in human studies of aging, exercise, orthopedic injury, knee osteoarthritis, chronic disease, and disuse ${ }^{1,2,3,4,5,6,7}$. However, methods 
to repeatedly and non-invasively analyze knee extensor muscle (quadriceps) strength in mechanistic rodent studies have been relatively limited. A method to determine in vivo quadriceps muscle contractility in rats was previously developed $^{8}$; however, extensive construction of noncommercially available equipment is required. Given the breadth of rodent models developed to study musculoskeletal outcomes following knee injury/osteoarthritis $9,10,11,12,13$ there exists a need for non-invasive assessment of quadriceps strength.

Furthermore, rodent studies investigating molecular mechanisms underpinning skeletal muscle adaptation often utilize mouse models due to the simplicity of genetic modification, as do many pharmacological intervention studies because of the decreased financial expense associated with lower weight-based dosing of a drug in mice compared to rats. We report a non-invasive method to repeatedly measure in vivo knee extensor function in the same mouse over time using commercially available equipment with minor modification, facilitating reproducibility among different laboratories, and providing more direct comparison to human strength outcomes.

\section{Protocol}

All experimental procedures were approved by University of Kentucky Institutional Animal Care and Use Committee.

\section{Equipment setup}

1. Confirm that machines are connected per manufacturer specifications.

2. If not already in place, attach the 300D-305C-FP motor with knee extension apparatus to the $809 \mathrm{C}$ animal platform.
3. Turn on the water pump to $37^{\circ} \mathrm{C}$ to begin heating the platform.

4. If computer is not already on, turn the computer on, followed by the High-Power Bi-Phase Stimulator and 2 Channel Dual-Mode Lever System.

5. Pour isoflurane into vaporizer to maximum fill line.

\section{Software setup}

1. Open the software (details provided in Table of Materials).

2. To use the Instant Stimulation feature in conjunction with Live Data Monitor to optimize probe placement (step 4), select Prepare Experiment followed by Configure Instant Stim (Figure 1). Set the Pulse Frequency $(\mathrm{Hz})$ as 125 , Pulse Width (ms) as 0.2 , Number of Pulses as 1, Train Frequency $(\mathrm{Hz})$ as 0.5 and Run Time (s) as 120.

3. Select File and open Live Data Monitor.

4. To perform twitch (step 5) and torque-frequency (Step 6) experiments, select a previously programmed study that includes appropriate twitch and knee extension torquefrequency experiments (detailed below in step 5 and step $6)$.

1. Select the appropriate experimental mouse or Add New Animal and input corresponding mouse information to be stored with torque data.

2. Select Next Experiment or Previous Experiment to transition from twitch protocol to torque-frequency sequence.

\section{Mouse setup}

1. Place individual mouse into the anesthetic chamber. 
2. Release oxygen tank valve and set oxygen flow rate at 1 $\mathrm{L} / \mathrm{min}$ with $2.5 \%$ isoflurane.

3. Ensure that mouse remains in the chamber with the lid securely closed until completely unconscious. Confirm complete loss of consciousness by absent foot reflex with toe pinch.

4. Place anesthetized mouse in a supine position with the head in the nosecone on the heated platform with oxygen flow rate at $1 \mathrm{~L} / \mathrm{min}$ with $2.5 \%$ isoflurane.

5. Shave hair from the right hind limb using electric clippers. Remove hair from the shaved area with an alcohol wipe and a small vacuum. Clean removed hair away from the hind limb and the platform.

6. Securely clamp the upper hind limb, posterior to the knee (Figure 2).

NOTE: Ensure that knee range of motion is not impeded.

7. Place the lower hind limb into the knee extension apparatus with the anterior tibia lightly touching the adjustable plastic piece (The Force In channel reading should read between 0 and $-1.0 \mathrm{mN}^{*} \mathrm{~m}$ ). Depending on the size of the lower hind limb of the mouse, surgical tape may be wrapped around the bottom portion of the adjustable plastic piece to allow the leg to rest securely. NOTE: Detailed images and dimensions of the customfabricated plastic piece are shown in Supplementary Figure 1.

8. Adjust knobs on platform to ensure the knee is bent at $60^{\circ}$.

9. Lightly place a piece of tape over the mouse torso onto the platform to prevent compensatory movement with maximal knee extension.

\section{Electrode placement}

1. Place electrodes subcutaneously $2-4 \mathrm{~mm}$ proximal to the knee directly above the quadriceps/knee extensor muscles (Figure 2). Electrodes should be approximately 1-2 mm apart.

2. To determine optimal placement of electrodes, utilize the Instant Stimulation function with Live Data Monitor. Set amperage/current at $50 \mathrm{~mA}$ for repeated twitches to confirm knee extension (the knee extensors will produce a negative twitch curve). Adjust the probes during Instant Stimulation to achieve maximal knee extension twitch torque as measured in the Live Data Monitor window.

NOTE: Figure 3 shows a representative Instant Stimulation output, confirming knee extension. Supplementary Video 1 and Supplementary Video 2 show real-time and slow-motion knee extensor twitches without the motor arm in place, allowing for visual confirmation of knee extension.

3. During repeated twitches with Instant Stimulation, palpate knee flexor muscles with the index finger to confirm no activation of antagonist muscles. To maximally stimulate knee extensors, probe repositioning may be necessary depending on body composition of the mouse and slight anatomical differences in the exact location of the motor point of the femoral nerve and knee extensor muscles.

NOTE: A muscle motor point is the location where the motor branch of a nerve enters the muscle belly and is the point with the least resistance to electrical conductivity and subsequently the highest responsiveness to electrical stimulation ${ }^{14,15}$. In clinical applications using electrical stimulation, this point is identified by scanning with a pen electrode to find 
the location above the muscle at which a muscle twitch occurs with the lowest injected current ${ }^{14,15}$. Identification of the muscle motor point is essential to facilitate optimal neuromuscular electrical stimulation ${ }^{15}$. In human clinical trials, muscle motor points for quadriceps muscles have been identified in the distal half of the muscle ${ }^{14}$. To achieve optimal knee extensor stimulation in mice, this technique was recapitulated using electrode placement with Instant Stimulation to most closely approximate muscle motor point locations typically found in the distal half of knee extensors. There exists some variability in electrode placement (from relatively superficial to deep) that results in maximal torque, and the Instant Stimulation function facilitates optimal electrode placement.

\section{Determination of optimal current}

1. Once optimal probe placement is determined, perform a series of progressive twitches to determine optimal amperage/current to be used for the torque-frequency experiment, with the goal of determining the lowest current to achieve the maximal twitch torque output. Begin with current set at $50 \mathrm{~mA}$ and select Run Experiment to produce a single twitch. Select Analyze Results to display torque output. Record the twitch torque displayed under Max Force with baseline subtracted.

NOTE: Select the option to invert the Force channel to convert measurements from negative torque to positive.

2. Increase the current to $60-70 \mathrm{~mA}$ and repeat twitch experiment. Record the twitch torque displayed under Max Force with baseline subtracted.
3. Continue with a series of twitch experiments in this manner (increasing approximately 10-20 mA with each progression) until twitch torque no longer increases (either plateaus or begins to decrease). Example of twitch series is shown in Table 1.

4. Record the lowest current at which the highest twitch torque was achieved. This current will be used and remain constant during the upcoming force-frequency experiment. Figure 4 shows a representative peak twitch.

\section{Torque-frequency experiment to determine peak isometric tetanic torque}

1. In the software (see Table of Materials), select the pre-programmed torque-frequency experiment for knee extension ensuring following setting. Stimulus duration: $0.35 \mathrm{~s}$, Frequency sequence: $10 \mathrm{~Hz}, 40 \mathrm{~Hz}, 120 \mathrm{~Hz}$, $150 \mathrm{~Hz}, 180 \mathrm{~Hz}, 200 \mathrm{~Hz}$, Rest period between pulses/ contractions: $120 \mathrm{~s}$

NOTE: Sampling rate is $10,000 \mathrm{~Hz}$ (default setting).

2. Run Experiment, Analyze Results, and manually record the torque displayed under Max Force with baseline subtracted (make sure the force channel is inverted, as knee extensor contraction will produce negative torque) at each frequency. Note the highest Max Force value as the peak isometric tetanic torque. Example of torque-frequency data is shown in Table 2 and Figure $\mathbf{5}$ shows a representative tetanus curve for the peak isometric tetanic torque output achieved at 120 $\mathrm{Hz}$.

\section{Termination of experiment}

1. Upon completion of the torque-frequency experiment, perform a follow up twitch and compare with the initial 
peak twitch at the same current to assess damage/ fatigue.

NOTE: In some models of injury and disease, increased fatigability of skeletal muscle is expected and does not constitute a problem with the experimental setup or the mouse.

2. When all torque measurements are completed, gently remove electrode probes, and unclamp the knee.

3. Turn off isoflurane and remove the mouse from the nose cone.

4. Place the mouse back into an appropriate cage placed on top of a warming pad. Monitor as the mouse recovers and regains consciousness.

NOTE: The mouse should be conscious and moving within 2-3 $\min$.

\section{Data analysis}

1. Extract data after the experiment from analysis software (see Table of Materials).

1. Open analysis software.

2. Select Get Data from the software.

3. Select Date on which experiment was performed and appropriate mouse code.

4. Select the frequency of interest (all twitch experiments and each frequency of the torquefrequency experiment will be listed).

5. Select Muscle Analysis.

6. Confirm that Use Baseline Correction is checked. NOTE: Baseline torque is calculated by the software as the average of the first 100 points sampled and subtracted from the absolute maximum torque value.
7. Record the torque value listed under Maximum. NOTE: Data presented here are unfiltered; however, a filter may be selected in the software, if desired.

2. Alternatively, as described above in step 6.2, manually record the torque output displayed under Max Force in real time at each torque-frequency point/contraction through the Analyze Results window.

1. Confirm that the baseline is subtracted, and force channel is inverted.

2. Input data into a spreadsheet for body weight normalization calculations (torque/body weight in grams) and graphing and statistical analyses of interest. Statistical software was used for the purpose of graphing torque-frequency curves and calculating area under the curve.

NOTE: Torque data are measured in $\mathrm{mN} . \mathrm{m}$ (milliNewton.meters).

3. To generate tetanus curves, export complete data from each frequency from the analysis software.

1. Repeat steps 8.1.1-8.1.4 above.

2. Select Export Data.

3. Select Raw Filtered Data and save in location of choice. MATLAB may be used to generate tetanus curves from the exported text file and/or for further analysis.

NOTE: MATLAB code to generate tetanus curve from text file is available upon request.

\section{Dual-mode lever system calibration}

1. Calibrate the system prior to the initial use to ensure accurate and reliable data, and repeat calibration 
periodically using the data collection software and known weights.

1. Open data collection software.

2. Click the Setup tab and select Channel Setup.

3. Select 305C-FP listed under My Instruments.

4. Click Calibrate Selected to open the Calibration Editor window.

5. To calibrate length, input a series of test voltages including both negative and positive voltages (e.g., $-3,-2,-1,0,1,2,3 \vee)$.

1. Click Set for the first line.

2. Click Read.

3. Measure the exact length of the lever arm in millimeters and input into the corresponding box.

4. Repeat for the next voltage.

5. Upon recording of all voltages, click Calculate Cal Factors (recorded in $\mathrm{mm} /$ volt).

6. To calibrate force in, utilize a set of known weights increasing in a linear progression.

1. Adjust the motor so that it is resting on the edge of the bench or table with the lever arm parallel to the benchtop and hanging over the edge to allow the weight to hang.

2. Hang the first weight from the lever arm using a rubber band. Under Applied Force, enter the known weight in grams accounting for the mass of the rubber band.

3. Select Read.

4. Repeat for at least 3 known weights.

\section{Select Calculate Cal Factor.}

6. To verify calculation, plot calibration data and curve fit by selecting Plot Cal.

7. To calibrate force out, enter calibration voltages (up to 10 volts)

1. Click Set directly next to the calibration voltage.

2. Repeat for each voltage line.

3. Gently apply pressure to the lever arm using a finger until the Force Out ceases to change and the motor arm begins to move.

4. Maintain this position. Select Read.

5. Repeat for each voltage line.

6. Select Calculate Cal Factor.

\section{Representative Results}

The torque-frequency curve utilizes lower frequencies to produce multiple isolated isometric twitches of relatively low torque and progresses through increasingly higher frequencies, resulting in fusion of twitches for an isometric tetanus contraction at which peak tetanic torque is obtained. The presented protocol for knee extension peak tetanic torque the force-frequency curve initiates at $10 \mathrm{~Hz}$ which elicits 3 isolated twitches. Partial fusion of twitches occurs at $40 \mathrm{~Hz}$, and peak tetanic torque is reached between $120-180$ $\mathrm{Hz}$ (Figure 5).

Figure 6 illustrates representative knee extension torquefrequency curves from female C57BL/6 mice. Three separate mice were tested at baseline, and the experiment was repeated in each mouse 2 weeks later for comparison to assess reproducibility. Torque-frequency curves are shown with raw torque values (Figure $\mathbf{6 A}$ ), as well as raw torque values normalized to mouse body weight (Figure 6B). 
Repeated observations demonstrate comparable results in all 3 mice with a 2-week rest period between experiments. Body weight normalized torque data should be considered in addition to raw torque, as minor fluctuation in weight may impact functional output and is not considered with raw torque alone. Furthermore, body weight normalized torque data facilitates comparison of mice of varying sizes. Torque can also be normalized to muscle wet weight or myofiber cross sectional area, as we have previously shown ${ }^{16}$.

Figure 7A shows the area under the curve using body weight normalized isometric torque data from complete torquefrequency experiments $(10 \mathrm{~Hz}, 40 \mathrm{~Hz}, 120 \mathrm{~Hz}, 150 \mathrm{~Hz}, 180$ $\mathrm{Hz}, 200 \mathrm{~Hz}$ ) for 4 separate C57BL/6 mice, highlighting similar total torque output and coefficients of variation between $5.6 \%$ to $8.8 \%$ with repeated experiments within the same mice. Data are most simply reported as peak tetanic torque (Figure 7B) which is the maximal torque value from the repeated tetanus isometric contractions from $120-200 \mathrm{~Hz}$. The peak tetanic torque output is comparable in 6-8-month-old female
C57BL/6 mice (Figure 7B) with coefficients of variation between $4.8 \%$ and $8.7 \%$ with longitudinal assessment within the same mice. Peak tetanic torque is most comparable to the gold-standard strength assessment in human studies: maximum isometric toque.

Furthermore, the knee extensor peak tetanic torque protocol is a useful tool to detect strength differences in multiple mouse models. Figure 8 demonstrates the stark contrast between knee extensor strength in a non-injured, healthy 6-month-old C57BL/6 female mouse (black line) and a transgenic mouse model of supraphysiological hypertrophy in which myostatin/ GDF8 is knocked out (blue line). We also show a peak tetanus curve from a C57BL/6 mouse 7 days after surgical transection of the anterior cruciate ligament (ACL-T) (red line), demonstrating a nearly $50 \%$ decline in peak torque after injury which is well outside the coefficients of variation observed with repeat testing of uninjured mice. Concurrent with human data ${ }^{17,18}$, strength is markedly diminished with ACL-T. All mice are female and of similar age (6-8 months).

\begin{tabular}{|c|c|c|}
\hline Twitch experiment & Amperage/Current (mA) & Torque (mN•m) \\
\hline 1 & 50 & 1.279 \\
\hline 2 & 70 & 1.341 \\
\hline 3 & 90 & 1.36 \\
\hline 4 & 110 & 1.362 \\
\hline 5 & $* 130$ & 1.449 \\
\hline 6 & 150 & 1.436 \\
\hline 7 & 140 & 1.333 \\
\hline
\end{tabular}

Table 1: Example of twitch series. * denotes optimal amperage/current. 


\begin{tabular}{|c|c|}
\hline Frequency $(\mathbf{H z})$ & Torque $(\mathbf{m N} \bullet \mathbf{m})$ \\
\hline 10 & 1.385 \\
\hline 40 & 1.869 \\
\hline 120 & $* \mathbf{1 8 . 7 6 5}$ \\
\hline 150 & 18.375 \\
\hline 180 & 17.97 \\
\hline 200 & 17.548 \\
\hline
\end{tabular}

Table 2: Example of torque-frequency curve data. * denotes peak tetanic torque.

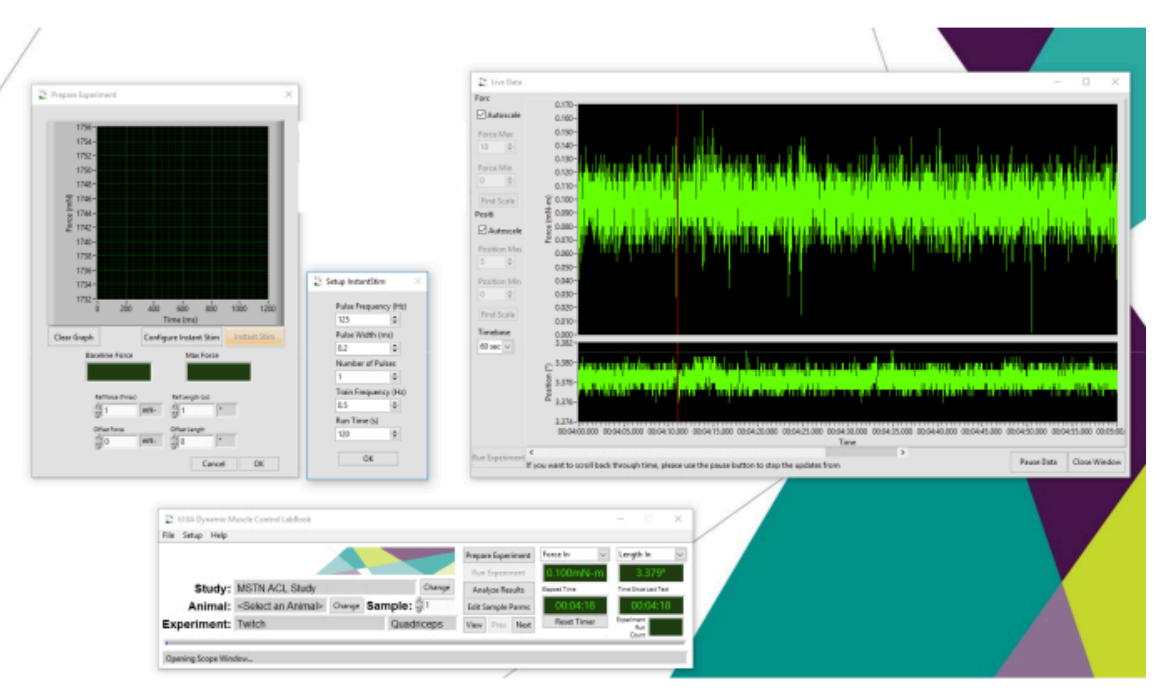

Figure 1: Data collection software setup. Illustration of setup for data collection software with Live Data Monitor. Please click here to view a larger version of this figure. 

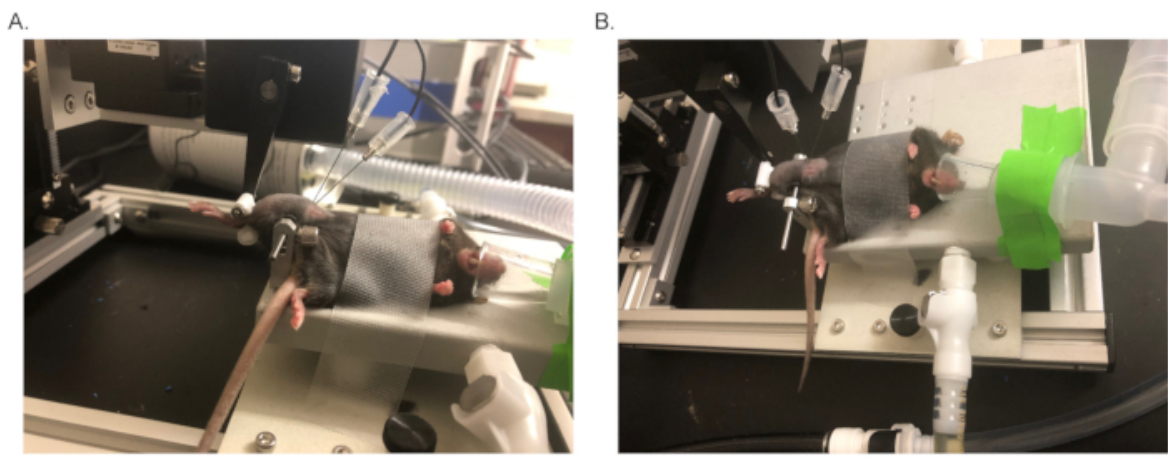

Figure 2: Mouse setup and electrode placement. (A-B) Supine position of the mouse receiving anesthesia via a nose cone on the heated platform. Upper hind limb is securely clamped, posterior to the knee to allow for unrestricted movement at the knee joint. Motor arm is adjusted so that knee is bent at approximately $60^{\circ}$. The femoral nerve motor point is stimulated by needle electrodes to activate contraction of knee extensors. Mouse setup is shown from a side view $(\mathbf{A})$ and overhead view (B). Please click here to view a larger version of this figure.

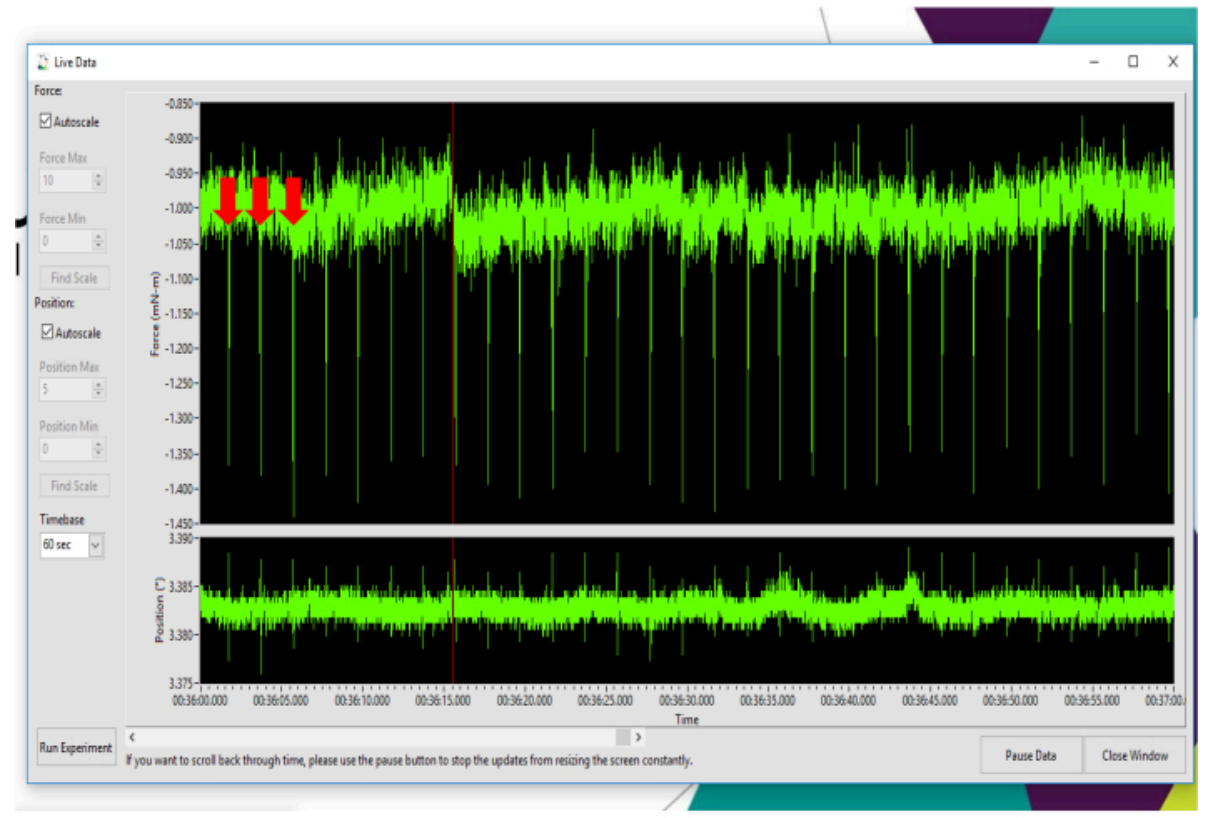

Figure 3: Determination of optimal electrode placement to achieve isometric knee extension. Representation of repeated negative twitches stimulated with $50 \mathrm{~mA}$ using the Instant Stimulation function and viewed in the Live Data Monitor. Red arrows indicate the first three knee extension twitches. Please click here to view a larger version of this figure. 


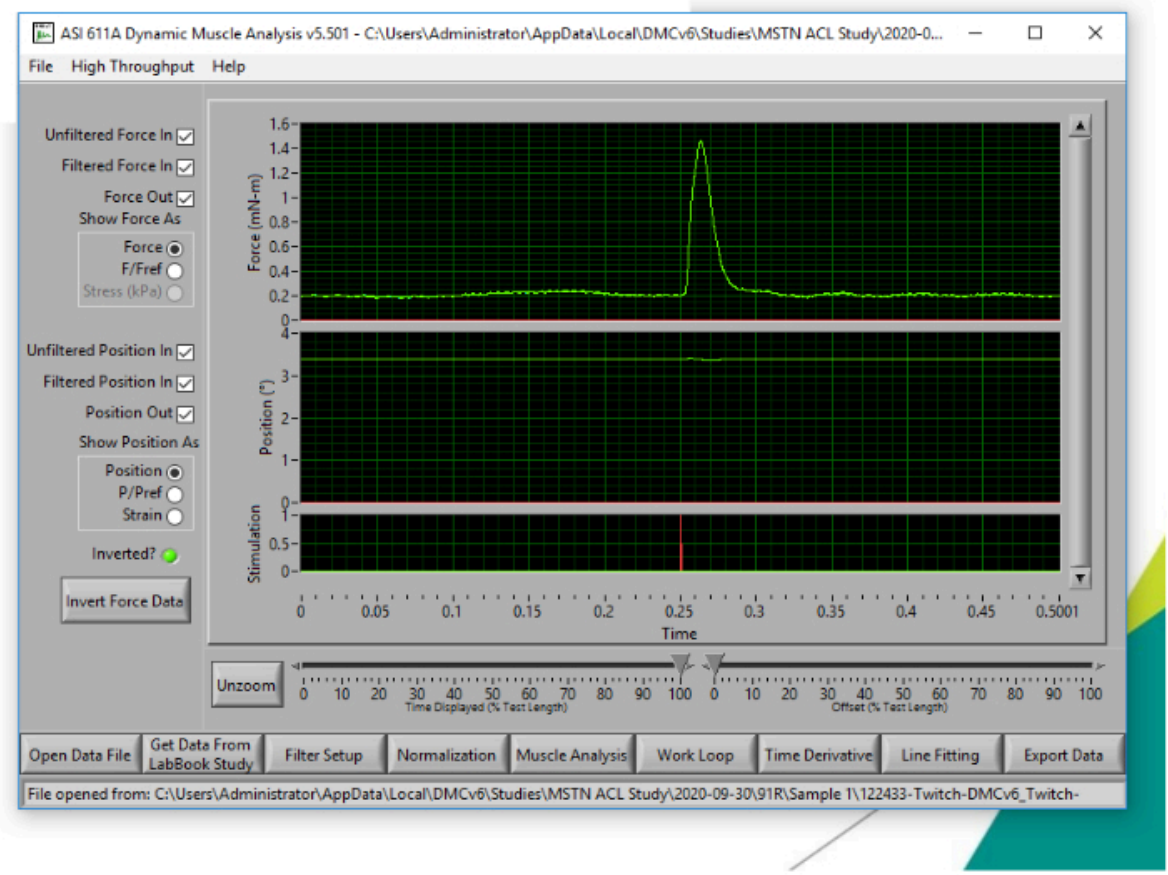

Figure 4: Representative twitch to determine optimal amperage. The lowest amperage to elicit the highest twitch isometric torque must be determined for the force-frequency experiment by repeated twitch experiments with progressively increased amperage. Please click here to view a larger version of this figure. 
A.

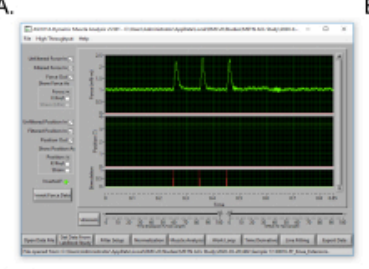

D.

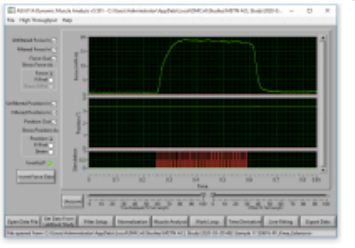

B.

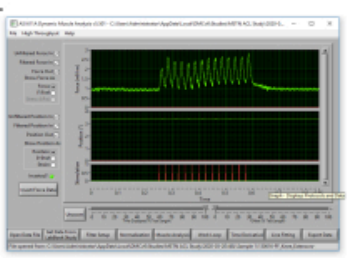

E.

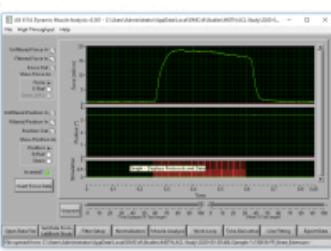

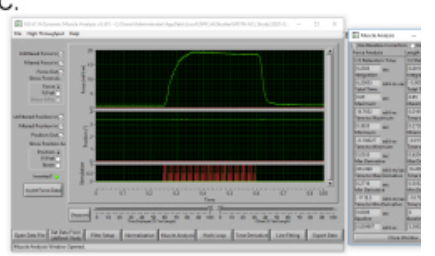

F.

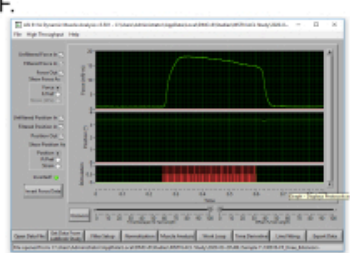

Figure 5: Representative tetanic torque curves throughout a torque-frequency experiment for the same mouse.

(A) Submaximal isometric tetanic torque produced at $10 \mathrm{~Hz}$. (B) Submaximal isometric tetanic torque at $40 \mathrm{~Hz}$. (C) Peak isometric tetanic torque output at $120 \mathrm{~Hz}$. (D) Isometric tetanic torque at $150 \mathrm{~Hz}$. (E) Isometric tetanic torque at $180 \mathrm{~Hz}$. (F) Isometric tetanic torque at $200 \mathrm{~Hz}$. Please click here to view a larger version of this figure.

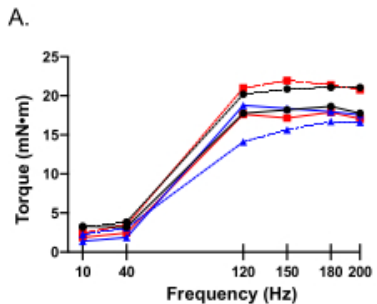

B.

- Mouse 1

- Mouse 1 repeat

- Mouse 2

$\rightarrow$ Mouse 2 repeat

- Mouse 3

- Mouse 3 repeal

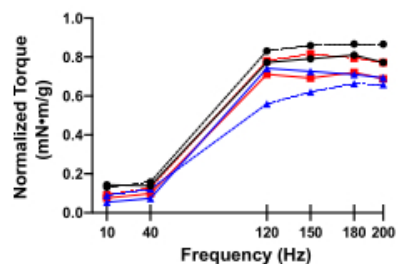

$\rightarrow$ Mouse 1

- Mouse 1 repeat

$\leftarrow$ Mouse 2

- Mouse 2 repeat

- Mouse 3

- Mouse 3 repeat

Figure 6: Representative torque-frequency curve data. (A-B). Torque-frequency curve at 2 different timepoints (week 1 and 3 ) in 3 separate mice, presented as raw peak torque (A) and raw peak torque normalized to body weight (B). Please click here to view a larger version of this figure. 
A.

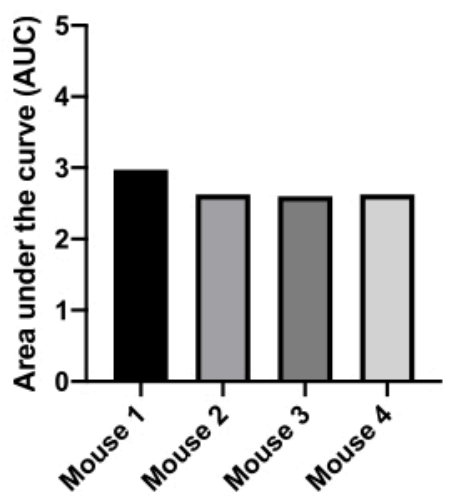

B.

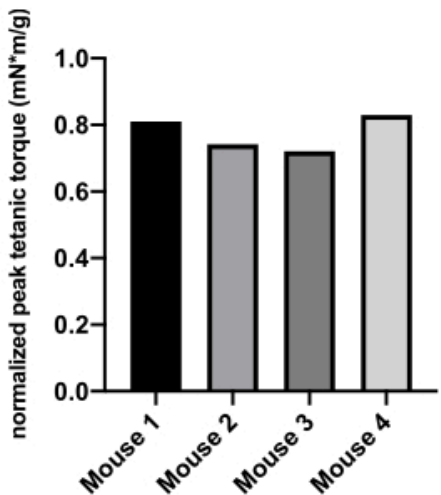

Figure 7: Representative area under the curve (AUC) and peak tetanic torque data. (A) AUC for 4 separate mice, presented as raw torque normalized to body weight. (B) Peak tetanic torque for the same 4 mice, presented as raw peak tetanic torque normalized to body weight. Please click here to view a larger version of this figure.

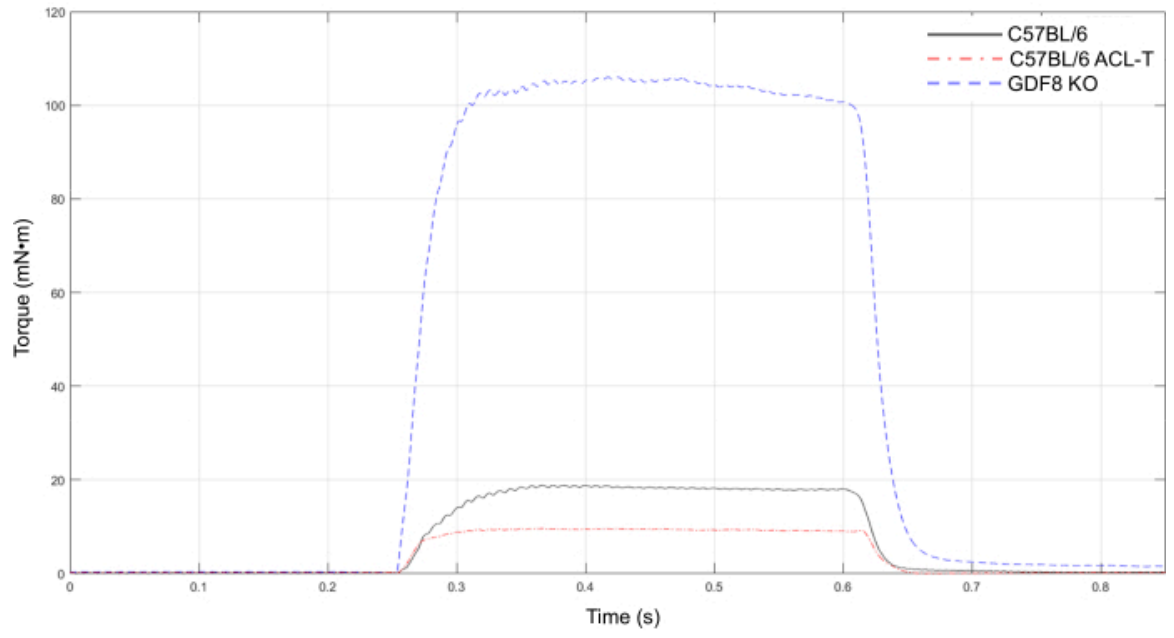

Figure 8: Peak tetanic torque of knee extensors in multiple mouse models. Representative peak torque tetanus curves for an overt hypertrophy transgenic mouse model (GDF8 KO), an uninjured healthy C57BL/6 mouse (mouse 2), and a C57BL/6 mouse 7 days after anterior cruciate ligament transection (ACL-T). Please click here to view a larger version of this figure. 
Supplementary Figure 1: Dimensions of custom fabricated plastic. Inset in red shows dimension of depth. Please click here to download this File.

\section{Supplementary Video 1: Real-time knee extensor twitch} without motor arm. Please click here to download this Video.

\section{Supplementary Video 2: Slow-motion knee extensor twitch without motor arm. Please click here to download} this Video.

\section{Discussion}

Measurement and analysis of muscle function in rodent models is imperative to make translational and meaningful inferences regarding histological and molecular skeletal muscle adaptations observed with exercise, injury, disease, and therapeutic treatment. We demonstrate a method to assess knee extensor maximal strength reliably and repeatedly in mice using commercially available equipment, with the adjustable plastic piece to hold the lower hind limb at the anterior tibia being the only custom fabricated part that may be replicated.

Common functional assessment tools have been widely used to repeatedly evaluate physical performance within the same mouse, such as treadmill running to volitional fatigue, rotarod performance test, inverted cling test, and grip strength test. However, while informative, these assessments involve cardiopulmonary and behavioral component(s), which can obfuscate the interrogation of neuromuscular function associated with these physical performance measures. Furthermore, elements of endurance, coordination, and balance are present in many of these functional assessments to varying levels, limiting clear interpretation relative to muscle strength. The force producing ability of rodent muscle(s) can be measured in vitro, in situ, or in vivo. Each approach has relative advantages and limitations. Specifically, with in vitro assessment, the muscle is completely isolated and removed from the body of the animal so that there is no influence from perfusion or innervation ${ }^{19}$. This yields a well-controlled environment to ascertain contractile ability but limits the size of the muscle being studied through dependency on passive diffusion of oxygen and nutrients during testing. In situ testing maintains the innervation and blood supply of the muscle, but is limited to a singular terminal assessment, as with in vitro testing ${ }^{20}$. Finally, in vivo testing is the least invasive with the muscle remaining in its native environment with percutaneous electrodes inserted near the motor nerve to electrically stimulate the muscle. A strength of the in vivo approach is the potential for longitudinal testing across time $21,22,23$.

In vivo evaluation of peak muscle contractility optimally measures maximal strength as the normal anatomy and physiology of the mouse remains intact and the method may be repeated on the same mouse before and after an intervention or throughout the lifespan. Specifically, in vivo measurement of knee extensor strength in mice is the murine strength assessment with the greatest translational relevance to human studies, as maximum knee extension torque is commonly measured and considered the goldstandard strength test in humans with correlation to various functional and health outcomes $24,25,26,27$. Moreover, knee extensor pathology is observed with aging as well as a myriad of injuries and diseases ${ }^{1,2,4,5,6}$, but assessing the impact of these conditions on knee extensor strength longitudinally in mice has not been readily attainable. 
Although this method offers utility to determine knee extensor peak torque in a longitudinal manner, certain limitations of the protocol should be considered. Lower frequencies between $40 \mathrm{~Hz}$ to $120 \mathrm{~Hz}$ were omitted from the torquefrequency protocol, which may limit the ability to detect left or rightward shifts in the torque-frequency curve with injury or disease. However, using this torque-frequency protocol, we have been able to detect alterations to peak tetanic torque in an ACL injury model and between C56BL/6 wild type mice and a transgenic mouse model of supraphysiological muscle mass (Figure 8). We note that it may be beneficial to secure the electrodes with helping hands or similar apparatus as muscle contractions may move electrodes slightly. We did not note any obvious displacement of electrodes with progressive contractions; however, the possibility of slight movement of the electrodes cannot be ruled out, which may impact muscle stimulation. Additionally, intramuscular electromyography (EMG) was not performed in conjunction with the stimulus protocol; however, inclusion of EMG measures may be feasible, if desired and appropriate for the experimental model of interest.

Assessment of knee extensor strength in murine models of orthopedic injury and disease will facilitate pre-clinical research with meaningful translational relevance to clinical strength measures. Our protocol enables precise and repeated assessment of maximal knee extensor strength in mice with commercially available equipment accessible to any laboratory.

\section{Disclosures}

Matthew Borkowski is employed by Aurora Scientific Inc., a company that may potentially benefit from the research results and is also an executive of the company.

\section{Acknowledgments}

We would like to thank Rosario Maroto for technical assistance. Research reported in this publication was supported by the National Institute of Arthritis and Musculoskeletal and Skin Diseases of the National Institutes of Health under Award Number R01 AR072061 (CSF). The content is solely the responsibility of the authors and does not necessarily represent the official views of the National Institutes of Health.

\section{References}

1. Brightwell, C. R. et al. Moderate-intensity aerobic exercise improves skeletal muscle quality in older adults. Translational Sports Medicine. 2 (3), 109-119 (2019).

2. Moro, T. et al. Muscle protein anabolic resistance to essential amino acids does not occur in healthy older adults before or after resistance exercise training. Journal of Nutrition. 148 (6), 900-909 (2018).

3. Angelozzi, M. et al. Rate of force development as an adjunctive outcome measure for return-to-sport decisions after anterior cruciate ligament reconstruction. Journal of Orthopedic Sports Physical Therapy. 42 (9), 772-780 (2012).

4. Kalyani, R. R. et al. Quadriceps strength, quadriceps power, and gait speed in older U.S. adults with diabetes mellitus: results from the National Health and Nutrition Examination Survey, 1999-2002. Journal of American Geriatric Society. 61 (5), 769-775 (2013).

5. Culvenor, A. G., Ruhdorfer, A., Juhl, C., Eckstein, F., Øiestad, B. E. Knee extensor strength and risk of structural, symptomatic, and functional decline in knee 
osteoarthritis: A systematic review and meta-analysis. Arthritis Care Res (Hoboken). 69 (5), 649-658 (2017).

6. Abramowitz, M. K. et al. Skeletal muscle fibrosis is associated with decreased muscle inflammation and weakness in patients with chronic kidney disease. American Journal of Physiology and Renal Physiology. 315 (6), F1658-F1669 (2018).

7. Arentson-Lantz, E. J., English, K. L., Paddon-Jones, D., Fry, C. S. Fourteen days of bed rest induces a decline in satellite cell content and robust atrophy of skeletal muscle fibers in middle-aged adults. Journal of Applied Physiology (1985). 120 (8), 965-975 (2016).

8. Pratt, S. J. P., Lovering, R. M. A stepwise procedure to test contractility and susceptibility to injury for the rodent quadriceps muscle. Journal of Biological Methods. 1 (2) (2014).

9. Kamekura, S. et al. Osteoarthritis development in novel experimental mouse models induced by knee joint instability. Osteoarthritis Cartilage. 13 (7), 632-641 (2005).

10. Kwok, J. et al. Histopathological analyses of murine menisci: implications for joint aging and osteoarthritis. Osteoarthritis Cartilage. 24 (4), 709-718 (2016).

11. Glasson, S. S., Blanchet, T. J., Morris, E. A. The surgical destabilization of the medial meniscus (DMM) model of osteoarthritis in the 129/SvEv mouse. Osteoarthritis Cartilage. 15 (9), 1061-1069 (2007).

12. Christiansen, B. A. et al. Musculoskeletal changes following non-invasive knee injury using a novel mouse model of post-traumatic osteoarthritis. Osteoarthritis Cartilage. 20 (7), 773-782 (2012).
13. Wurtzel, C. N. et al. Pharmacological inhibition of myostatin protects against skeletal muscle atrophy and weakness after anterior cruciate ligament tear. Journal of Orthopedic Research. 35(11), 2499-2505 (2017).

14. Botter, A. et al. Atlas of the muscle motor points for the lower limb: implications for electrical stimulation procedures and electrode positioning. European Journal of Applied Physiology. 111 (10), 2461-2471 (2011).

15. Gobbo, M., Maffiuletti, N. A., Orizio, C., Minetto, M. A. Muscle moter point identification is essential for optimizing neuromuscular electrical stimulation use. Journal of Neuroengineering and Rehabililitation. 11, 17 (2014).

16. Neelakantan, $\mathrm{H}$. et al. Small molecule nicotinamide Nmethyltransferase inhibitor activates senescent muscle stem cells and improves regenerative capacity of aged skeletal muscle. Biochemical Pharmacology. 163, 481-492 (2019).

17. Kline, P. W., Morgan, K. D., Johnson, D. L., Ireland, M. L., Noehren, B. Impaired quadriceps rate of torque development and knee mechanics after anterior cruciate ligament reconstruction with patellar tendon autograft. American Journal of Sports Medicine. 43 (10), 2553-2558 (2015).

18. Hiemstra, L. A., Webber, S., MacDonald, P. B., Kriellaars, D. J. Knee strength deficits after hamstring tendon and patellar tendon anterior cruciate ligament reconstruction. Medicine and Science in Sports and Exercise. 32 (8), 1472-1479 (2000).

19. Park, K. H. et al. Ex vivo assessment of contractility, fatigability and alternans in isolated skeletal muscles. Journal of Visualized Experiments. (69), e4198 (2012). 
20. Maclntosh, B. R., Esau, S. P., Holash, R. J., Fletcher, J. R. Procedures for rat in situ skeletal muscle contractile properties. Journal of Visualized Experiments. (56), e3167 (2011).

21. Chiu, C. S. et al. Non-invasive muscle contraction assay to study rodent models of sarcopenia. BMC Musculoskeletal Disorder. 12246 (2011).

22. Mintz, E. L., Passipieri, J. A., Lovell, D. Y., Christ, G. J. Applications of in vivo functional testing of the rat tibialis anterior for evaluating tissue engineered skeletal muscle repair. Journal of Visualized Experiments. (116), e 54487, (2016).

23. Gerlinger-Romero, F. et al. Non-invasive assessment of dorsiflexor muscle function in mice. Journal of Visualized Experiments. (143), e58696, (2019).

24. Davis, C. C., Ellis, T. J., Amesur, A. K., Hewett, T. E., Di Stasi, S. Improvements in knee extension strength are associated with improvements in self-reported hip function following arthroscopy for femoroacetabular impingement syndrome. International Journal of Sports Physical Therapy. 11 (7), 1065-1075 (2016).

25. Omori, G. et al. Quadriceps muscle strength and its relationship to radiographic knee osteoarthritis in Japanese elderly. Journal of Orthopedic Science. 18 (4), 536-542 (2013).

26. Wilk, K. E., Romaniello, W. T., Soscia, S. M., Arrigo, C. A., Andrews, J. R. The relationship between subjective knee scores, isokinetic testing, and functional testing in the ACL-reconstructed knee. Journal of Orthopedic Sports and Physical Therapy. 20 (2), 60-73 (1994).

27. Bobowik, P., Wiszomirska, I. Diagnostic dependence of muscle strength measurements and the risk of falls in the elderly. Internation Journal of Rehabilitation Research. 43 (4), 330-336 (2020). 